indicate that there are social and cultural obstacles facing Indochinese refugees in Japan, especially learning the Japanese language.

Using data from the 1986 census, Hugo assesses Vietnamese settlers' adjustment and well-being after almost a decade of resettlement in Australia. Evidence indicates that they are in many ways similar to other groups of immigrants from Eastern Europe, Southern Europe and the Middle East during their initial years of settlement. Although there appears to be astable and thriving community established shortly after Vietnamese refugees settled in Australia, unemployment and underemployment among them are higher when compared with other Australians. The lack of recognition of their qualifications, an inability to speak English, the current economic recession, a declining demand for blue-collar workers (especially in the unskilled sectors), and an undercurrent of racism, are factors contributing to the disadvantaged and marginalized position of Vietnamese in Australia.

Issues-such as the necessity of disentangling the complexities of root causes; policy responses of first countries of asylum; the effect of deterrence, detention and the determination process on asylum seekers in camps; and the subsequent resettlement process-will certainly be debated and examined by scholars in refugee studies. This Special Issue lends theoretical and empirical insights for further investigations. For example, in a time of globalization and burden sharing, how and why should the consistency of Singapore's refugee policy be objectively assessed? What valuable lessons have been learned and are applicable to the international community in their concerted effort to provide protection to asylum seekers? What kinds of relief programs would provide appropriate and adequate assistance to asylum seekers? This Special Issue is essential reading for those interested in the boat people phenomenon, as well as the wider scope of refugee studies.

Lawrence Lam is an Associate Director (Education) at the Centre for Refugee Studies.

\section{IMMIGRATION AND REFUGEE BOARD STATISTICS ${ }^{1}$}

\begin{tabular}{|c|c|c|c|c|c|c|}
\hline \multicolumn{7}{|c|}{ Table 1: Regional Summary (January - June 1992) } \\
\hline \multicolumn{7}{|c|}{ INITIAL HEARING STAGE (Credible Basis) } \\
\hline $\begin{array}{l}\text { Claims concluded } \\
\text { Withdrawn/abandoned } \\
\text { Decisions rendered }\end{array}$ & $\begin{array}{r}\text { antic } \\
279 \\
1 \\
278\end{array}$ & $\begin{array}{r}\text { Que. } \\
6,381 \\
32 \\
6,349\end{array}$ & $\begin{array}{r}\text { Ont. } \\
9,545 \\
86 \\
9,459\end{array}$ & $\begin{array}{r}\text { Prairies } \\
346 \\
20 \\
326\end{array}$ & $\begin{array}{r}\text { B.C. } \\
878 \\
7 \\
871\end{array}$ & $\begin{array}{r}\text { National } \\
17,429 \\
146 \\
17,283\end{array}$ \\
\hline $\begin{array}{l}\text { Of these decisions } \\
\text { Claims rejected: } \\
\text { Eligibility } \\
\text { Credible basis } \\
\text { To full hearing }\end{array}$ & $\begin{array}{r}0 \\
9 \\
269\end{array}$ & $\begin{array}{r}20 \\
147 \\
6,182\end{array}$ & $\begin{array}{r}19 \\
479 \\
8,961\end{array}$ & $\begin{array}{r}14 \\
58 \\
254\end{array}$ & $\begin{array}{r}8 \\
44 \\
819\end{array}$ & $\begin{array}{r}61 \\
737 \\
16,485\end{array}$ \\
\hline \multicolumn{7}{|l|}{ FULL HEARING STAGE } \\
\hline $\begin{array}{l}\text { Claims heard to } \\
\text { completion } \\
\text { (include 1989-92 cases) }\end{array}$ & 256 & 5,543 & 8,424 & 277 & 779 & 15,279 \\
\hline $\begin{array}{l}\text { Decisions rendered } \\
\text { Claims rejected } \\
\text { Claims upheld }\end{array}$ & $\begin{array}{l}255 \\
111 \\
144\end{array}$ & $\begin{array}{l}5,397 \\
1,858 \\
3,539\end{array}$ & $\begin{array}{l}8,445 \\
2,845 \\
5,600\end{array}$ & $\begin{array}{r}287 \\
87 \\
200\end{array}$ & $\begin{array}{l}751 \\
524 \\
227\end{array}$ & $\begin{array}{r}15,135 \\
5,425 \\
9,710\end{array}$ \\
\hline $\begin{array}{l}\text { Withdrawn/abandoned } \\
\text { Decisions pending* } \\
\text { Claims pending }\end{array}$ & $\begin{array}{r}26 \\
46 \\
219\end{array}$ & $\begin{array}{r}340 \\
733 \\
5,028\end{array}$ & $\begin{array}{r}404 \\
1,269 \\
7,210\end{array}$ & $\begin{array}{r}9 \\
19 \\
139\end{array}$ & $\begin{array}{r}74 \\
211 \\
1,007\end{array}$ & $\begin{array}{r}853 \\
2,278 \\
13,603\end{array}$ \\
\hline \multicolumn{7}{|c|}{$\begin{array}{l}\text { "Decisions pending include all claims heard to completion since January } 1,1989 \text { for which no } \\
\text { decision had been rendered by the end of the reporting period. } \\
\text { " Claims pending include all claims referred to the CRDD full hearing stage, that have not been } \\
\text { finalized (i.e. by a positive or negative decision or by withdrawal or abandonment) as of the } \\
\text { end of the reporting period. }\end{array}$} \\
\hline
\end{tabular}

\section{Table 2}

Acceptance Rates* for Refugee Claimants from Top-ten Source Countries (IRB Statistics Period: Jan. - June, 1992)

\begin{tabular}{|c|c|c|c|c|c|c|c|c|c|c|}
\hline \multicolumn{5}{|c|}{ Credible Basis Level Processing } & \multicolumn{5}{|c|}{ Full Hearing Level Processing } & \multirow{3}{*}{$\begin{array}{c}\text { Overall } \\
\text { Accept.\% } \\
96.0\end{array}$} \\
\hline Country $\operatorname{Pr}$ & rocesse & Yes & No & Rate\% & Processed & Wdwn & Yes & No & Rate\% & \\
\hline Sri Lanka 3 & 3,164 & 3,155 & 9 & 99.7 & 2,779 & 35 & 2,650 & 102 & 96.3 & \\
\hline Somalia & 1,929 & 1,924 & 5 & 99.7 & 2,056 & 45 & 1,910 & 200 & 90.5 & 90.3 \\
\hline USSR & 776 & 749 & 22 & 97.1 & 748 & 70 & 422 & 313 & 57.4 & 55.7 \\
\hline China & 76 & 11 & 36 & 70.0 & ו & 17 & 167 & 710 & 19.0 & 0.0 \\
\hline Iran & 718 & 711 & 2 & 99.3 & 666 & 38 & 554 & 109 & 83.6 & 829 \\
\hline Pakistan & 705 & 682 & 18 & 97.4 & 470 & 20 & 278 & 188 & 59.7 & 57.4 \\
\hline El Salvador & r 671 & 63 & 29 & 95.5 & 670 & 57 & 234 & 356 & 39.7 & 37.7 \\
\hline Lebanon & 579 & 573 & 2 & 99.1 & 476 & 27 & 233 & 194 & 54.6 & 53.9 \\
\hline Yugoslavia & 531 & 504 & 20 & 70.0 & 300 & 20 & 149 & 88 & 62.9 & 57.1 \\
\hline Ghana & 421 & 374 & 39 & 90.6 & 493 & 59 & 157 & 377 & 29.4 & 27.4 \\
\hline
\end{tabular}

("Acceptance rates for individual countries are based on adjudicated claims only; withdrawn [Wdwn] claims are not included.) Charts: ASA

${ }^{1}$ Immigration and Refugee Board news release dated August 20, 1992. 
Refuge

York Lanes Press

Centre for Refugee Studies

Suite 351, York Lanes, York University $\mathbf{4 7 0 0}$ Keele Street, North York

Ontario, Canada M3J 1 P3

Phone: (416) 736-5843 - Fax: (416) 736-5837

Electronic Mail via Bitnet Address: REFUGE @YORKVM1

Postage Paid in Toronto, Canada Second Class Mail Registration No. 5512 Return Postage Guaranteed

\section{CONFERENCE}

